\title{
Digging deeper into platform game level design: session size and sequential features
}

\author{
Noor Shaker, Georgios N. Yannakakis and Julian Togelius \\ IT University of Copenhagen, Rued Langaards Vej 7, 2300 Copenhagen, Denmark \\ \{nosh, yannakakis, juto\}@itu.dk
}

\begin{abstract}
A recent trend within computational intelligence and games research is to investigate how to affect video game players' in-game experience by designing and/or modifying aspects of game content. Analysing the relationship between game content, player behaviour and self-reported affective states constitutes an important step towards understanding game experience and constructing effective game adaptation mechanisms. This papers reports on further refinement of a method to understand this relationship by analysing data collected from players, building models that predict player experience and analysing what features of game and player data predict player affect best. We analyse data from players playing 780 pairs of short game sessions of the platform game Super Mario Bros, investigate the impact of the session size and what part of the level that has the major affect on player experience. Several types of features are explored, including item frequencies and patterns extracted through frequent sequence mining.
\end{abstract}

\section{Introduction}

What makes a good computer game? What features should be presented in the game, where in the game should they be presented, how often and in which order? What features should be manipulated to alter specific player experience? And what is the minimum length of time a player need to play in order to elicit a particular affective state? We describe a method that we believe can be used to help answer these questions, and exemplify it with an investigation based on data from hundreds of players playing Super Mario Bros. In the process, we arrive at tentative partial answers to these questions in the context of Super Mario Bros levels.

Many analyses of computer games can be found in the literature, both in terms of game mechanics and from a player perspective based on how the player can interact with the game. For example, some researchers have analysed game content into its constituent parts, or "design patterns" [1], [5], [17]; others have tried to state general facts about what makes games enjoyable [9], [8]. Most of this research, however, tackles this problem from a top-down perspective, creating theories of player experience based on qualitative methods. Some attempts have been made to construct computational models form qualitative theories [23], [26].

Another direction that is related to this work is the procedural generation of game content (PCG) with no or limited human designer input. PCG has recently received increasing attention with the use of artificial and computational intelligence methods to generate different aspects of game content such as maps [3], [21], levels [18], [11] and 


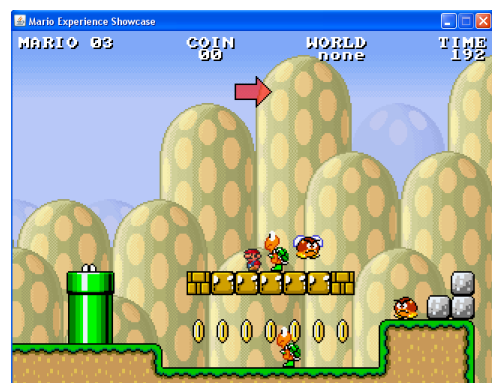

Fig. 1. Snapshot from Infinite Mario Bros, showing Mario standing on horizontally placed boxes surrounded by different types of enemies

racing tracks [4], [20]. One interesting direction in PCG is the online generation of personalized game content [6], [7], [16]. One approach towards achieving this goal is first to model the relationship between player experience and game content. This requires the construction of data-driven models based on data collected about the game, the player behaviour and correlating this data with data annotated with player experience tags [25].

This paper continues our previous work on player experience modeling in a version of Super Mario Bros [10], [12], [14]. The main focus of those experiments was on modelling the relationship between direct features of game content, players' playing styles and reported players experience by using features extracted from the full game sessions. The dataset used for those studies constitutes of 480 game pairs constructed using four different controllable features. In a recent paper [15] we reported preliminary explorations of predicting player experience of engagement based only on level features and introduced the use of sequence mining to extract simple patterns from game content, using an incomplete version of a larger and more detailed dataset. We also explored constructing models from parts of levels in order to find the minimum segment length which would allow us to perform meaningful adaptation.

In this paper, we explore the full dataset of 780 game pairs played by hundreds of players. We draw upon the approach proposed in [15] and we extend it in through (1) investigating the three emotional states; engagement frustration and challenge; (2) constructing player experience models based on game content, player behaviour and reported player experience; (3) investigating the impact of the size of game session on the accuracy of predicting players' reported emotion; (4) analysing the importance of the features for each emotional state with respect to their relative placement within the game and (5) exploring direct and sequential feature representations.

The testbed platform game we are using for this study is a modified version of Markus "Notch" Persson's Infinite Mario Bros. The game is well known and the benchmark software has been used relatively extensively as a testbed for research [22], [12], [13], [2], [14] and for the Mario AI Championship [16]. Please refer to [22] for details about the game and the gameplay experience it provides.

The paper is organized as follows. Section 2 explains the process followed to collect data from players. Section 3 presents the two forms that have been used to represent the 
collected data. A method that has been used to mine sequential data is presented in Section 4. Section 5 describes player experience modeling via preference learning. The process of segmenting the levels into smaller chucks and constructing models based on the segments is discussed in Section 6, while Section 7 presents the experiments conducted and the analysis of the results. Finally, Section 8 presents our conclusions.

\section{Experiment Design}

The following section describes the level generation process, the survey that has been designed to collect the data and the types of data that were extracted. The level generator of the game has been modified to generate levels according to the following six controllable features

- The number of gaps in the level, $G$ and the average with of gaps, $\bar{G}_{w}$.

- The number of enemies, $E$.

- Enemies placement, $E_{p}$. The way enemies are placed around the level is determined by three probabilities which sum to one; on or under a set of horizontal blocks, $P_{x}$; within a close distance to the edge of a gap, $P_{g}$ and randomly placed on a flat space on the ground, $P_{r}$.

- The number of powerups, $N_{w}$.

- The number of boxes, $B$. We define one variable to specify the number of the different types of boxes that exist; blocks and rocks. Blocks (which look like squares with question marks) contain hidden elements such as coins or powerups. Rocks (which look like squares of bricks) may hide a coin, a powerup or simply be empty. The generator randomly select one of these two types for each box generated.

The selection of these particular controllable features was done with the intent to cover the features that have the most impact on the investigated affective states. The placement of gaps, powerups and boxes is approximately uniformly random. Two states (low and high) are set for each of the controllable parameters above except for enemies placement which has been assigned three different states allowing more control over the difficulty and diversity of the generated levels. For example, setting $P_{g}$ to $80 \%$ results in a level with a majority of enemies placed around gaps, increasing the level difficulty. Other features of the level have been assigned fixed values. For example, the number of gaps in the level can be either two or six, while the number of free coins is fixed to seven in all generated levels. The level generator constructs level by exploring the total number of pairwise combinations of these states (96). This number can be reduced to 40 by analysing the dependencies between these features and eliminating the combinations that contain independent variables.

\subsection{Data collection}

The game survey study [15] has been designed to collect subjective affective reports expressed as pairwise preferences of subjects playing the different levels of the game by following the experimental protocol proposed in [28]. The game sessions have been 
Table 1. Gameplay features extracted from data recorded during gameplay

\begin{tabular}{ccl}
\hline Category & Feature & Description \\
\hline Time & $t_{\text {comp }}$ & Completion time \\
& $t_{\text {last } \text { aft }}$ & Playing duration of last life over total time spent on the level \\
& $t_{\text {duck }}$ & Time spent ducking (\%) \\
& $t_{\text {jump }}$ & Time spent jumping (\%) \\
& $t_{\text {left }}$ & Time spent moving left $(\%)$ \\
& $t_{\text {right }}$ & Time spent moving right (\%) \\
& $t_{\text {run }}$ & Time spent running $(\%)$ \\
& $t_{\text {small }}$ & Time spent in Small Mario mode (\%) \\
& $t_{\text {big }}$ & Time spent in Big Mario mode (\%) \\
\hline Interaction & $n_{\text {coins }}$ & Free coins collected (\%) \\
with items & $n_{\text {coin Blocks }}$ & Coin blocks pressed or coin rocks destroyed (\%) \\
& $n_{\text {powerups }}$ & Powerups pressed (\%) \\
& $n_{\text {boxes }}$ & Sum of all blocks and rocks pressed or destroyed (\%) \\
\hline Interaction & $k_{\text {cannonFlower }}$ & Times the player kills a cannonball or a flower (\%) \\
with enemies & $k_{\text {goombaKoopa }}$ & Times the player kills a goomba or a koopa (\%) \\
& $k_{\text {stomp }}$ & Opponents died from stomping (\%) \\
& $k_{\text {unleash }}$ & Opponents died from unleashing a turtle shell (\%) \\
\hline Death & $d_{\text {total }}$ & Total number of deaths \\
& $d_{\text {cause }}$ & Cause of the last death \\
Miscellaneous & $n_{\text {mode }}$ & Number of times the player shifted the mode (Small, Big, Fire) \\
& $n_{\text {jump }}$ & Number of times the jump button was pressed \\
& $n_{\text {gJump }}$ & Difference between the number of gaps and the number of jumps \\
& $n_{\text {duck }}$ & Number of times the duck button was pressed \\
& $n_{\text {state }}$ & Number of times the player changed the state between: \\
& & standing still, run, jump, moving left, and moving right \\
\hline
\end{tabular}

constructed using a level width of 100 Super Mario Bros units (blocks) based on all combinations of the controllable features. 780 pairs of games (exhausting the space of controllable features) were played by hundreds of players. Participants' age covers a range between 16 and 64 years from different origins. Complete games were logged enabling complete replays. The following types of data were extracted.

- Gameplay Data: All player actions and interactions with game items and their corresponding time-stamps have been recorded with the full trajectory of Mario.

- Reported Player Experience: A 4-alternative forced choice questionnaire is presented to the players after playing each pair asking them to report their emotional preferences across three user states: engagement, challenge and frustration. The selection of these states is based on earlier game survey studies [12] and our intention to capture both affective and cognitive components of gameplay experience [25].

\section{Data Representation}

\subsection{Direct Features}

Several features have been directly extracted from the data recorded during gameplay. Most of these features appear in our previous studies [10], [14] and the choice of them is made in order to be able to represent the difference between a large variety of Super Mario Bros playing styles. These features are presented in Table 1. 


\subsection{Sequential Features}

We investigate another form of data representation that allows including features based on ordering in space or time by means of sequences. Sequences of game content and players' behaviour yields patterns that might be directly linked to player experience. These patterns provide a mean for an in-depth analyses of the relationship between the player and the game. There are several possible approaches to generate sequences from interaction logs. In this paper we concentrate on two types of sequences:

- Content corresponding to gameplay events: Game content at the specific player position is recorded whenever the player performs an action or interacts with game items. Different content events are used: increase/decrease in platform height, $P^{\uparrow} / P^{\downarrow}$; existence of an enemy, $P_{e}$; existence of a coin, block or rock, $P_{d}$; existence of a coin, block or rock with an enemy, $P_{e d}$; and the beginning/ending of a gap $P_{g s} / P_{g e}$.

- Sequential Gameplay Features: Sequences representing different players' behaviour have been generated by recording key pressed/released events (action event). The list of events that have been considered includes: pressing an arrow key to move right, left, or duck $(\bullet, \varangle, \mathbf{\nabla})$; pressing the jump key, ; ; pressing the jump key in combination with right or left key $(\Uparrow, \Uparrow)$; pressing the run key in combination with right or left key $\left(R^{\star}, R^{\triangleleft}\right)$; pressing the run and jump keys in combination with right/left ( $R^{\boldsymbol{}}$, $R^{\triangleleft \Uparrow}$ ); and not pressing any key, $S$.

\section{Sequence Mining}

Sequence mining techniques have been applied to extract useful information from the different types of the sequences generated. Generalised Sequential Pattern (GSP) algorithm [19] has been used to mine the sequences and find frequent patterns within the dataset of sequences. The GSP algorithm has been chosen because of its advantages over other apriori-based sequence mining algorithms. Using GSP, we can discover patterns with a predefined minimum support, min $_{\text {sup }}$ ( the minimum number of times a pattern has to occur in the data-sequences to be considered frequent), and specify a time constraints within which adjacent events can be considered elements of the same pattern, $\max _{\text {gap }}$. Different $\min _{\text {sup }}$ values have been explored to obtain a reasonable trade off between considering patterns that are generalised over all players and more specific patterns. For the experiments presented in this paper, we use a $\min _{\text {sup }}$ of 500 which forces a sequence pattern to occur in at least $31.8 \%$ of the samples to be considered frequent. The $\max _{\text {gap }}$ has a great impact on the number of frequent patterns that can be extracted. By assigning a large value to this parameter, we allow more generalised patterns to be taken into account. The experiments conducted for tuning the value of this parameter showed that a max $_{g a p}$ of 1 second provides a good trade off between the number of patterns extracted and their expressiveness value. Different sequence length values have been explored, the experiments showed that the number of extracted subsequences is quite large for sequences containing information about players' behaviour. In order to lower the feature space dimensionality and the computational cost of searching for relevant features we chose to use only frequent sequences of length three. 


\section{Preference Learning for Modelling Playing Experience}

Neuroevolutionary preference learning has been used in order to construct models that approximate the function between gameplay features, controllable features, and reported affective preferences. We start the models' constructing procedure by selecting the relevant subset of features for predicting each emotional state, this is done by using Sequential Forward Selection (SFS) to generate the input vector of single-layer perceptrons (SLPs) [27]. The feature subset derived from SFS using SLP is then used as the input to small multi-layer perceptron (MLP) models containing one hidden layer of two neurons. The quality of a feature subset and the performance of each MLP is obtained through the average classification accuracy in three independent runs using 3-fold cross validation across five runs. Parameter tuning tests have been conducted to set up the parameters' values for neuroevolutionary user preference learning that yield the highest accuracy and minimise computational effort. A population of 100 individuals is used, and evolution run for 20 generations. A probabilistic rank-based selection scheme is used, with higher ranked individuals having higher probability of being chosen as parents. Finally, reproduction was performed via uniform crossover, followed by Gaussian mutation of $1 \%$ probability.

\section{Level Segmentation}

The purpose of segmenting the level is to draw a picture of the importance of the features with respect to player experience; different features correlated with player experience for each emotional state could be extracted from each segment of the game pointing out to positions in the games where these features play a role in triggering particular affective state. By segmenting the levels we can also identify the size of the level segment that generates the best prediction accuracy of the three emotional states. That segment size can then potentially be used to set the frequency of a real-time adaptation mechanism for maximising particular player experience (as in [24], [14]).

We follow the same process presented in [15] for segmenting the level into half and one-third width segments but instead of using the same feature set and calculating each feature value across all segments, we run SFS to select the most relevant feature subset from all direct and sequential features for each segment across all emotional states.

\section{Experiments and Analysis}

We ran a number of experiments to select features from the full levels and from different segments into which the levels have been divided. Player experience models were constructed based on the different subset of features selected from direct and sequential features for each segment across the three emotional states. Table 2 presents the selected features and the models' accuracies.

The networks found vary in the number of selected features and performance. The most accurate model is the one for predicting challenge (91.23\%) with a large subset of 13 features selected from the full levels. Engagement comes next with a best model accuracy of $86.43 \%$ obtained from features extracted from the first segment out of two 
Table 2. The features selected from the set of direct and sequential features for predicting engagement, frustration and challenge using sequential feature selection with SLP and simple MLP models and the corresponding models' performance

\begin{tabular}{|c|c|c|c|c|c|c|}
\hline & Full level & $1^{\text {st }} \mathrm{seg} / 2$ & $2^{\text {nd }} \mathrm{seg} / 2$ & $1^{s t} \operatorname{seg} / 3$ & $2^{n d} \operatorname{seg} / 3$ & $3^{r d} \mathrm{seg} / 3$ \\
\hline \multicolumn{7}{|c|}{ Engagement } \\
\hline $\begin{array}{l}\text { Selected } \\
\text { features }\end{array}$ & $\begin{array}{c}t_{\text {comp }} \\
n_{\text {coins }} \\
d_{\text {cause }} \\
t_{\text {small }} \\
t_{\text {jump }} \\
E \\
n_{\text {coinBlocks }} \\
t_{\text {big }} \\
t_{\text {run }} \\
n_{\text {jump }} \\
P^{\downarrow} P_{g s} P_{g e} \\
\end{array}$ & $\begin{array}{c}n_{\text {state }} \\
t_{\text {big }} \\
d_{\text {cause }} \\
t_{\text {right }} \\
E \\
S \Uparrow \Uparrow \\
S_{\Uparrow \Uparrow \Uparrow}\end{array}$ & 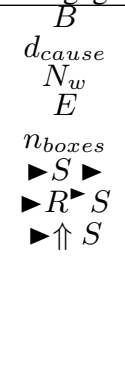 & $\begin{array}{c}n_{\text {gJump }} \\
t_{\text {left }} \\
n_{\text {boxes }} \\
B \\
\bar{G}_{w} \\
d_{\text {cause }} \\
E_{p} \\
n_{\text {coins }} \\
\nabla^{\bullet} S \\
\mathrm{~S} \triangleleft \mathrm{S}\end{array}$ & $\begin{array}{c}t_{\text {right }} \\
E \\
n_{\text {gJump }} \\
d_{\text {cause }} \\
t_{\text {duck }} \\
G\end{array}$ & $\begin{array}{c}B \\
\mathrm{G} \\
t_{\text {jump }} \\
d_{\text {cause }} \\
k_{\text {unleash }}\end{array}$ \\
\hline$M L P_{\text {perf }}$ & $75.21 \%$ & $86.43 \%$ & $72.03 \%$ & $72.19 \%$ & $71.69 \%$ & $72.86 \%$ \\
\hline \multicolumn{7}{|c|}{ Frustration } \\
\hline $\begin{array}{l}\text { Selected } \\
\text { features }\end{array}$ & $\begin{array}{c}t_{\text {right }} \\
d_{\text {total }} \\
d_{\text {cause }} \\
t_{\text {lastLift }} \\
\bar{G}_{w} \\
G \\
n_{\text {jump }} \\
P_{d} P_{d} P^{\uparrow} \\
\Uparrow\end{array}$ & $\begin{array}{c}n_{\text {gJump }} \\
G \\
n_{\text {coins }} \\
E \\
t_{\text {left }} \\
R^{\bullet} \\
R^{\triangleright} R^{-\Uparrow}\end{array}$ & $\begin{array}{c}G \\
n_{g J u m p} \\
\bar{G}_{w} \\
d_{\text {cause }} \\
k_{\text {stomp }}\end{array}$ & $\begin{array}{c}n_{\text {jump }} \\
t_{\text {small }} \\
t_{\text {left }} \\
S \uparrow^{-} S\end{array}$ & $\begin{array}{c}G \\
B \\
t_{\text {small }}\end{array}$ & $\begin{array}{c}E_{p}^{\prime} \\
k_{\text {goombaKoopa }} \\
B \\
G \\
n_{\text {gJump }} \\
t_{\text {left }} \\
t_{\text {right }} \\
S S \\
R \bullet R-R^{-\Uparrow} S\end{array}$ \\
\hline$M L P_{\text {perf }}$ & $85.88 \%$ & $81.73 \%$ & $79.85 \%$ & $78.72 \%$ & $78.15 \%$ & $73.45 \%$ \\
\hline \multicolumn{7}{|c|}{ Challenge } \\
\hline $\begin{array}{l}\text { Selected } \\
\text { features }\end{array}$ & $\begin{array}{c}t_{\text {lastLift }} \\
n_{\text {jump }} \\
d_{\text {total }} \\
n_{\text {coins }} \\
t_{\text {right }} \\
\bar{G}_{w} \\
E_{p} \\
t_{\text {left }} \\
k_{\text {stomp }} \\
P_{d} P_{d} P_{d} \\
P^{\downarrow} P_{g s} P_{g e} \\
\Uparrow{ }^{\top} S \\
P^{\uparrow} P^{\downarrow} P^{\downarrow}\end{array}$ & $\begin{array}{c}t_{\text {right }} \\
n_{\text {gJump }} \\
n_{\text {state }} \\
G \\
t_{\text {small }} \\
B \\
\text { - } S\end{array}$ & $\begin{array}{c}G \\
B \\
d_{\text {cause }} \\
n_{\text {gJump }}\end{array}$ & $\begin{array}{c}n_{\text {gJump }} \\
n_{\text {boxes }} \\
n_{\text {powerups }} \\
t_{\text {right }} \\
n_{\text {mode }} \\
t_{\text {left }} \\
t_{\text {big }}\end{array}$ & $\begin{array}{c}G \\
E \\
k_{\text {cannonFlower }} \\
n_{\text {jump }} \\
k_{\text {unleash }} \\
\Uparrow S\end{array}$ & $\begin{array}{c}E_{p} \\
k_{\text {stomp }} \stackrel{ }{B} \\
E \\
k_{\text {unleash }} \\
>S S\end{array}$ \\
\hline$M L P_{\text {Perf }}$ & $91.23 \%$ & $75.6 \%$ & $77.19 \%$ & $72.41 \%$ & $73.52 \%$ & $69.38 \%$ \\
\hline
\end{tabular}


followed by frustration which can be predicted with an accuracy up to $85.88 \%$ from a subset of ten features extracted from the full level.

Segmenting the sessions resulted in a performance increase for the models of predicting engagement while a performance decrease has been observed for the experience models of frustration and challenge. The model constructed based on features selected from the first half of the session for predicting engagement significantly (significant effect is determined by $p<0.01$ over 10 runs in this paper) outperforms all other models constructed on full and other partial information. A significant performance decrease was found for predicting frustration when constructing the models based on features extracted from segments with one third of the full size. Using features from the full sessions, we were able to predict challenge with high accuracy that is significantly better than all other models constructed on partial information.

The results suggest that different sizes of game session are needed to elicit different affective states. While challenge can be predicted with high accuracy from the full sessions, smaller session size somewhat count-intuitively appears to give better results for predicting engagement, and frustration can be predicted with high accuracy from full and half size sessions.

The different subsets of features selected from each segment draw a picture of the importance of the positioning of the features within the game and the different impacts this has on the different emotional states under investigation. Some content features have been selected from the full sessions and also appear in the subset of features selected from the parts, such as the number of enemies $(E)$ and the number of gaps $(G)$ for predicting engagement and frustration respectively. This suggests the importance of these features for eliciting a particular emotional state regardless of their specific positioning within the game. Other features like the number of powerups $\left(N_{w}\right)$ appears to have an impact on engagement when presented in the second half of the game. This can be explained by the fact that powerups are more important to the players towards the end of the game since this increases their chance of winning, the selection of cause of death feature in all segments also supports this assumption. It's worth noting that only one controllable feature has been selected for the best model for predicting engagement and the rest of the features relate to the particular playing style for each player. Most aspects of level design appear to have a large impact on challenge since five content features (direct and sequential) have been selected for the best model of predicting challenge.

\section{Discussion and Conclusion}

In this paper, we reported on the creation of data-driven computational models that predict three players' reported affective states based on level design and gameplay features. We investigated direct and sequential features of both game content and gameplay, as well as the impact of session sizes. We were able to predict players' reported levels of engagement, frustration and challenge with high accuracy.

Our previous study [15] concluded that segmenting the levels yields a performance decrease when constructing models based only on game content features. The results presented in this paper show that different session sizes should be considered for investigating the different emotional states when gameplay data is also considered as input 
to the models. Challenge can be best predicted with longer sessions' size than the ones needed for predicting frustration or engagement. The results indicate that the models performance in general significantly decreases when segmenting the session into more than two segments. This suggests that segmenting the data into more than two segments causes information loss. Another possible explanation is that the session size should be longer than a particular length to elicit a specific emotional state, and it appears that one third of the level size is too small to consider the reported player experience valid while the gameplay experience and the reported affects can still be considered valid for one half of the session size for engagement and frustration.

The different subsets of features selected for predicting affective states suggest differing relative importance of design elements for different aspects of player experience. This has the potential to partly decouple dissimilar aspects of player adaptation.

The results presented here will feed into our ongoing research on modelling player affect and preferences in Super Mario Bros, with the ultimate goal of producing an effectively player-adaptive version of the game, but could also inform separate studies.

\section{Acknowledgments}

The research was supported in part by the Danish Research Agency, Ministry of Science, Technology and Innovation; project "AGameComIn" (274-09-0083).

\section{References}

1. Björk, S., Holopainen, J.: Patterns in game design. Cengage Learning (2005)

2. Bojarski, S., Congdon, C.: Realm: A rule-based evolutionary computation agent that learns to play mario. In: Computational Intelligence and Games (CIG), 2010 IEEE Symposium. pp. 83-90 (2010)

3. Cardamone, L., Yannakakis, G., Togelius, J., Lanzi, P.: Evolving interesting maps for a first person shooter. Applications of Evolutionary Computation pp. 63-72 (2011)

4. Cardamone, L., Loiacono, D., Lanzi, P.L.: Interactive evolution for the procedural generation of tracks in a high-end racing game. Interface pp. 395-402 (2011)

5. Hullett, K., Whitehead, J.: Design patterns in fps levels. In: FDG '10: Proceedings of the Fifth International Conference on the Foundations of Digital Games. pp. 78-85. ACM, New York, NY, USA (2010)

6. Jennings-Teats, M., Smith, G., Wardrip-Fruin, N.: Polymorph: dynamic difficulty adjustment through level generation. In: Proceedings of the 2010 Workshop on Procedural Content Generation in Games. pp. 11:1-11:4. PCGames '10, ACM, New York, NY, USA (2010)

7. Kazmi, S., Palmer, I.: Action recognition for support of adaptive gameplay: A case study of a first person shooter. International Journal of Computer Games Technology 2010, 1 (2010)

8. Koster, R.: A theory of fun for game design. Paraglyph press (2004)

9. Malone, T.: What makes computer games fun? (abstract only). In: Proceedings of the joint conference on Easier and more productive use of computer systems. (Part - II): Human interface and the user interface - Volume 1981. p. 143. CHI '81, ACM, New York, NY, USA (1981)

10. Pedersen, C., Togelius, J., Yannakakis, G.N.: Modeling player experience in super mario bros. In: CIG'09: Proceedings of the 5th international conference on Computational Intelligence and Games. pp. 132-139. IEEE Press, Piscataway, NJ, USA (2009) 
11. Pedersen, C., Togelius, J., Yannakakis, G.N.: Modeling player experience for content creation. IEEE Transactions on Computational Intelligence and AI in Games 2(1), 54-67 (2010)

12. Pedersen, C., Togelius, J., Yannakakis, G.N.: Modeling player experience for content creation. IEEE Transactions on Computational Intelligence and AI in Games 2(1), 54-67 (2010)

13. Perez, D., Nicolau, M., O'Neill, M., Brabazon, A.: Evolving behaviour trees for the mario ai competition using grammatical evolution. In: Applications of Evolutionary Computation, Lecture Notes in Computer Science, vol. 6624, pp. 123-132. Springer Berlin / Heidelberg (2011)

14. Shaker, N., Yannakakis, G.N., Togelius, J.: Towards Automatic Personalized Content Generation for Platform Games. In: Proceedings of the AAAI Conference on Artificial Intelligence and Interactive Digital Entertainment (AIIDE). AAAI Press (October 2010)

15. Shaker, N., Yannakakis, G.N., Togelius, J.: Feature Analysis for Modeling Game Content Quality. In: IEEE Transactions on Computational Intelligence and AI in Games (CIG) (2011)

16. Shaker, N., Togelius, J., Yannakakis, G.N., Weber, B., Shimizu, T., Hashiyama, T., Sorenson, N., Pasquier, P., Mawhorter, P., Takahashi, G., Smith, G., Baumgarten, R.: The 2010 Mario AI championship: Level generation track. IEEE Transactions on Computational Intelligence and Games (2011)

17. Smith, G., Cha, M., Whitehead, J.: A framework for analysis of $2 d$ platformer levels. In: Sandbox '08: Proceedings of the 2008 ACM SIGGRAPH symposium on Video games. pp. 75-80. ACM, New York, NY, USA (2008)

18. Smith, G., Whitehead, J., Mateas, M.: Tanagra: A mixed-initiative level design tool. In: Proceedings of the International Conference on the Foundations of Digital Games (2010)

19. Srikant, R., Agrawal, R.: Mining sequential patterns: Generalizations and performance improvements. Advances in Database TechnologyEDBT'96 pp. 1-17 (1996)

20. Togelius, J., De Nardi, R., Lucas, S.: Towards automatic personalised content creation for racing games. In: Computational Intelligence and Games, 2007. CIG 2007. IEEE Symposium on. pp. 252-259. IEEE (2007)

21. Togelius, J., Preuss, M., Yannakakis, G.: Towards multiobjective procedural map generation. In: Proceedings of the 2010 Workshop on Procedural Content Generation in Games. p. 3. $\operatorname{ACM}(2010)$

22. Togelius, J., Karakovskiy, S., Koutník, J., Schmidhuber, J.: Super mario evolution. In: Proceedings of the 5th international conference on Computational Intelligence and Games. pp. 156-161. CIG'09, IEEE Press, Piscataway, NJ, USA (2009), http://dl.acm.org/citation.cfm?id=1719293.1719326

23. Tognetti, S., Garbarino, M., Bonanno, A., Matteucci, M.: Modeling enjoyment preference from physiological responses in a car racing game. IEEE Transactions on Computational Intelligence and AI in Games (2010)

24. Yannakakis, G.N., Hallam, J.: Real-time Game Adaptation for Optimizing Player Satisfaction. IEEE Transactions on Computational Intelligence and AI in Games 1(2), 121-133 (June 2009)

25. Yannakakis, G.N., Togelius, J.: Experience-Driven Procedural Content Generation. IEEE Transactions on Affective Computing (2011)

26. Yannakakis, G., Hallam, J.: A generic approach for generating interesting interactive pacman opponents. In: In Proceedings of the IEEE Symposium on Computational Intelligence and Games. pp. 94-101 (2005)

27. Yannakakis, G.N., Hallam, J.: Entertainment modeling through physiology in physical play. Int. J. Hum.-Comput. Stud. 66, 741-755 (October 2008), http://dl.acm.org/citation.cfm?id=1410473.1410682

28. Yannakakis, G.N., Maragoudakis, M., Hallam, J.: Preference learning for cognitive modeling: a case study on entertainment preferences. Trans. Sys. Man Cyber. Part A 39, 1165-1175 (November 2009), http://dx.doi.org/10.1109/TSMCA.2009.2028152 